\title{
EXPERIMENTAL ANALYSIS OF A PEM FUEL CELL PERFORMANCE AT VARIABLE LOAD WITH ANODIC EXHAUST MANAGEMENT OPTIMIZATION
}

\author{
Bruno Belvedere, Michele Bianchi, Alberto Borghetti, \\ Andrea De Pascale, Mario Paolone, Roberta Vecci \\ Faculty of Engineering, University of Bologna \\ viale del Risorgimento, 2- 40136 - Bologna, Italy
}

\begin{abstract}
In this paper an investigation on the performance of a commercial Proton Exchange Membrane (PEM) fuel cell, tested at the laboratories of the University of Bologna, is carried out. The investigation takes into account the management of anodic exhaust and relevant effects on the flooding phenomenon. To address the problem of flooding, it is necessary to run periodically the purge process of the fuel cell (FC) resulting into an overall decrease of the efficiency of the transformation process. This operation is performed by opening the so-called Outlet Control Valve (OCV) located along the anodic exhaust line. The aim of this analysis is to optimize the purge process to: (i) increase the FC lifetime, (ii) reduce the amount of hydrogen that is discharged with water and (iii) increase the FC efficiency. An investigation on the benefits in terms of fuel utilization factor and costs, resulting from optimization of the FC purge process, has been analyzed.
\end{abstract}

\section{INTRODUCTION}

Flooding (accumulation of excess water in a PEM) can happen at both the cathode and anode side of the FC membrane (1). Low $\mathrm{H}_{2}$ flow rates and current densities, water injected for cooling, humidification with conditions of low temperatures and water back-diffusion phenomenon, from cathode to anode promoted by a low hydration state of the fuel gas stream, leads to flooding (2-5). The FC stack rated performance in terms of output DC current and voltage can be significantly diminished by the flooding phenomenon due to a sudden increase of concentration losses. Impurities can be deposited on the catalyst and transported in the membrane replacing $\mathrm{H}^{+}$ions. Consequently, the electrodes performances decrease and the conductivity is reduced over the time, leading to FC failure $(2,4)$. To remove excess water and impurities inside the FC, it is necessary to operate a periodic purging.

\section{THE EXPERIMENTALTEST BENCH}

The experimental test bench is a micro-grid composed of the following components: (i) a $4.5 \mathrm{~kW} \mathrm{H}$ fuelled PEM-FC stack; (ii) an electrochemical energy storage system coupled with a common AC bus by means of a bidirectional inverter; (iii) a connection with external power sources; (iv) a load emulator subsystem; (v) an electric board connecting the power sources inverters to the load emulator. This micro-grid is designed to operate in island mode, but also connected to the external electric network, as described more in detail in previous works by the authors $(6,7)$. Figure 1

illustrates the supply circuits of fuel (in red) where, at the anode outlet side, the Outlet Control Valve (OCV) is used, a valve operating in dead-end mode, i.e. accomplishing a periodic purging of the water ( $\mathrm{FC}$ reaction product migrating in the anode compartment) and of the accumulating impurities.

\section{THE PURGE PROCESS ANALISYS}

The purge process has a fundamental role on the stability of the power supplied by the FC. Mokmeli et al. (5) showed that the amount and the concentration of impurities in the FC increases with time in the absence of purge. Due to this increasing accumulation of impurities, the stack is characterized by voltage drop increase over time. For this reason the purge process should be periodically run in a FC energy system. To operate the purging, the dead-end valve was installed downstream of the FC stack. The purge occurs during all the time in which the OCV is open. During this time, also a quantity of fuel is discharged and thus it is not used to realize the FC electrochemical reactions.

The two main control parameters of the periodic purge process and of the OCV are (Figure 2): the time between two consecutive purges, named here $T_{f l}$, and the purge time, $T_{p u}$. the $T_{f l}$ is equal to

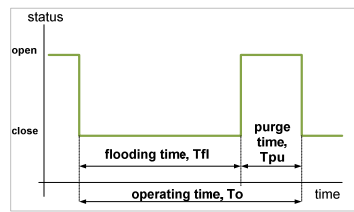

Figure 2 the time interval in which the OCV is closed and thus water accumulates in the FC anodic side, causing flooding; the $\mathrm{Tt}_{\mathrm{pu}}$ is the time during which the OCV is open and thus impurities, inert gases and excess water are eliminated out of the FC.

A programming logic for the purge process is typically based on the programming of these two parameters as a function of time. This purge control approach is based on the setting of the OCV characteristic times, independently on the FC operating conditions in terms of current. A simplified way to realize the 
purging process using this approach is to do it assuming constant values for both $T_{p u}$ and $T_{f l}$. The values of these two parameters should be chosen as a tradeoff between the minimization of amount of discharged hydrogen and of the voltage loss of the FC, resulting in an improvement in terms of fuel utilization. Finally, the purge can be operated when the stack voltage equals a predetermined threshold value, with the aim to protect and preserve the longevity and performance of the FC. The amount of purge time must be such as to allow recovery only of voltage loss.

\section{THE FC PURGE PROGRAMMING LOGIC: ANALYSIS}

The purge programming logic currently implemented in the PEM-FC is a function of time. The original values of the $T_{f l}$ and the $T_{p u}$ were set by the manufacturer and are respectively $20 \mathrm{~s}$ and $2.5 \mathrm{~s}$, kept constant for each FC power output value. This purge process is optimized for the maximum FC power, but it is very inefficient for low and medium FC power. This is due to considerable unreacted $\mathrm{H}_{2}$ loss during the opening time of the $\mathrm{OCV}$. To reduce the amount of unreacted $\mathrm{H}_{2}$ during the purge and at the same time safeguarding the FC internal components, it is necessary to improve the implemented purge logic, by optimizing the purge process. The aim of this purge process optimization is to increase the fuel utilization factor, $U_{f}$, defined as the ratio between the theoretical fuel energy, necessary to bring about the electrochemical reactions, and the introduced fuel chemical energy. The increase of $U_{f}$ leads also to an increase of the FC efficiency, $\eta$. To optimize the purge process a new programming logic has been adopted to investigate the $T_{\mathrm{ff}}$. In this new logic the values of purge parameters differ according to the FC delivered power. In particular, the $T_{f l}$ of the purges at different FC power levels, is studied imposing a maximum permissible $\mathrm{FC}$ voltage drop, $\Delta \mathrm{V}_{\max }$, and keeping constant the $\mathrm{T}_{\mathrm{pu}}$, equal to $2.5 \mathrm{~s}$. The maximum permissible voltage loss value $(0.65 \mathrm{~V})$ corresponds to the actual FC voltage drop during the flooding phase at FC maximum power. The calculation of $T_{f l}$ using this new logic is performed assuming a linear trend of the FC voltage versus time (Figure 3). For each of the examined FC power levels, the optimized flooding time was obtained solving equation: $\alpha T_{f l}+\Delta V_{\max }=0$ where $\Delta V_{\text {max }}$ : is the value of maximum allowable $\mathrm{FC}$ voltage loss, $(0.65 \mathrm{~V}) ; \alpha$ is the slope of straight line with which the FC

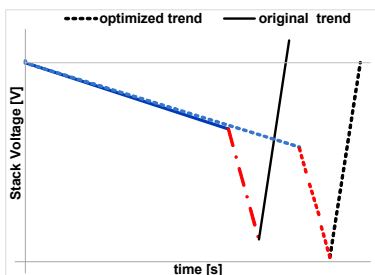

Figure 3 voltage behavior is estimated during the flooding

Table 1 shows the mean input power $\left(\mathrm{P}_{\mathrm{M}}\right)$ with reference to the fuel LHV, the mean $\mathrm{H}_{2}$ mass flow rate $\left(\mathrm{m}_{\mathrm{H} 2}\right)$ and the $\mathrm{FC}$

\begin{tabular}{|c|c|c|c|c|c|c|c|}
\hline \multirow{2}{*}{$\begin{array}{c}\text { FC } \\
\text { Power } \\
\text { P }_{\mathrm{FC}} \\
{[\mathrm{W}]}\end{array}$} & \multicolumn{3}{|c|}{ Flooding Time $=20 \mathrm{~s}$} & \multicolumn{3}{|c|}{ Optimized Flooding Time } & \multirow[b]{2}{*}{$\begin{array}{c}\Delta \mathrm{E}_{\max } \\
{[\%]}\end{array}$} \\
\hline & $P_{M}[W]$ & $\begin{array}{c}\mathbf{m}_{\mathrm{H} 2} \\
{[\mathbf{k g} / \mathbf{s}]} \\
\cdot 10^{-5}\end{array}$ & $\eta$ & $\mathrm{P}_{\mathrm{M}}{ }^{+}[\mathrm{W}]$ & $\begin{array}{c}\mathrm{m}_{\mathrm{H}^{+}} \\
{[\mathrm{kg} / \mathrm{s}]} \\
\cdot 10^{-5}\end{array}$ & $\eta^{+}$ & \\
\hline 500 & 2273 & 1.890 & 0.22 & 2942 & 2.452 & 0.29 & 30 \\
\hline 1500 & 4167 & 3.472 & 0.36 & 4688 & 3.906 & 0.40 & 12.9 \\
\hline 2500 & 6579 & 5.482 & 0.38 & 7576 & 6.313 & 0.42 & 7.6 \\
\hline 3500 & 8975 & 7.478 & 0.39 & 9210 & 7.676 & 0.40 & 2.85 \\
\hline 4500 & 11840 & 9.867 & 0.38 & 11840 & 9.867 & 0.38 & 0 \\
\hline
\end{tabular}

efficiency $(\eta)$ obtained using the original purge programming logic and the new one (values with the superscript + ), for each of the FC power levels analyzed. FC efficiency versus mean fuel inlet power (Figure 4) increases using the optimization of the purge process (dotted red line). In particular, this benefit occurs at low and medium FC power, where the actual purge logic provided lower performance. The savings of fuel energy introduced into the FC (Table 1), obtained using the new purge programming logic, can be evaluated using the index: $\Delta E=1-\left\lfloor E^{+} / E\right\rfloor$ where $E=\int_{0}^{\tau} m \cdot L H V \cdot d t ; E^{+}=\int_{0}^{\tau} m^{+} \cdot L H V \cdot d t$

$E$ is the fuel input energy introduced into the FC during the time $\tau$ in which the FC delivers the same power (this time period was chosen sufficiently long to include a high number of purges), evaluated according to the actual purge programming logic; $\mathrm{E}^{+}$is the input energy in case of the new purge

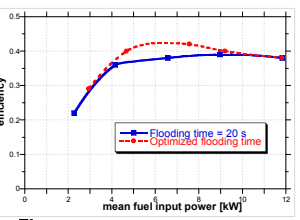

Figure 4 logic. Figure 5 shows the change of the fuel utilization factor using both currently purge logic $\left(U_{f}\right)$ and new one $\left(U_{f}^{+}\right)$.

\section{CONCLUSIONS}

In this paper an analysis on the purge process at the anode side of the PEM and the purge operation that allows to recover the instantaneous FC voltage drop has been carried out. The purge process has been analyzed in different FC power levels. For each of these power levels, the optimized purge has been obtained by changing the flooding time. To overcome the inefficiency of the actual purge programming logic implemented in the PEM-FC at the low and medium powers, the optimization of purge process has been carried out. A new purge programming logic, characterized by a flooding time variable and a purge time constant has been tested. Using this new logic the FC efficiency increases significantly, especially at medium power levels, obtaining a saving in terms of input fuel energy.

\section{NOMENCLATURE}

\begin{tabular}{ll} 
Symbols \\
\hline$E$ fuel energy ref.to LHV, [kJ] & $\mathrm{T}_{\mathrm{o}}$ operating time, [s] \\
$m \quad \mathrm{H}_{2}$ mass flow rate, [kg/s] & $\mathrm{T}_{\mathrm{pu}}$ purge time, [s] \\
$\mathrm{P}_{\mathrm{FC}} \mathrm{FC}$ power level, [W] & $\mathrm{Uf}$ fuel utilization factor, [-] \\
$\mathrm{T}_{\mathrm{fl}}$ flooding time, [s] & $\mathrm{V}$ voltage [V]
\end{tabular}

\section{REFERENCES}

(1) G. Hinds, NPL Report DEPC-MPE 002, 2004,pp.25-42.

(2) J. Gou, P. Pei, Y. Wang, The dynamic behaviour of pressure during purge process on the anode of a PEM fuel cell, Power Sources

(3) D. A. McKay, W.T. Ott, A. G. Stefanopoulou, Modeling, parameter identification, and validation of reactant water dynamics for a fuel cell stack, Proceeding of the ASME IMECE 2005.

(4) J.St-Pierre, D. P. Wilkinson, S. Knights, M. Bos, J. New Mat. Electrochem. Syst. 3 (2000) 99-106.

(5) A. Mokmeli, S. Asghari, An investigation into the effect of anode purging on the fuel cell performance, Int, Jour. of Hydrogen Energy.

(6) B. Belvedere, M. Bianchi, A. De Pascale, Experimental analysis of a cogenerative performance of a PEM fuel cell based energy system, Proceedings of ICAE, 16-18 May 2011 - Perugia, Italy

(7) B. Belvedere, M. Bianchi, A. Borghetti, M. Paolone, Design, implementation and testing of an automatic power management system for residential stand-alone microgrid with power supply, preprints of the $18^{\text {th }}$ IFAC World Congress , Milano (Italy), 2011. 